\title{
BMJ Open Organisation and function of the primary motor cortex in chronic pain: protocol for a systematic review and meta-analysis
}

\author{
Wei-Ju Chang, ${ }^{1}$ Neil E O'Connell, ${ }^{2}$ Emma Burns, ${ }^{1}$ Lucy S Chipchase, ${ }^{1}$ \\ Matthew B Liston, ${ }^{1}$ Siobhan M Schabrun ${ }^{1}$
}

To cite: Chang W-J

O'Connell NE, Burns E, et al. Organisation and function of the primary motor cortex in chronic pain: protocol for a systematic review and metaanalysis. BMJ Open 2015;5: e008540. doi:10.1136/ bmjopen-2015-008540

- Prepublication history and additional material is available. To view please visit the journal (http://dx.doi.org/ 10.1136/bmjopen-2015008540).

Received 20 April 2015 Revised 26 August 2015 Accepted 2 September 2015

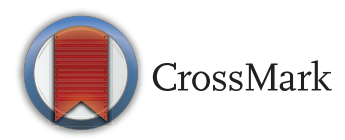

\footnotetext{
${ }^{1}$ University of Western Sydney, School of Science and Health, Penrith, New South Wales, Australia ${ }^{2}$ Department of Clinical Sciences, Health Economics Research Group, Institute of Environment, Health and Societies, Brunel University, Uxbridge, UK
}

Correspondence to Dr Siobhan M Schabrun; s.schabrun@uws.edu.au

\begin{abstract}
Introduction: Primary motor cortical (M1) adaptation in the form of altered organisation and function is hypothesised to underpin motor dysfunction observed in chronic pain. The aim of this review is to assess the evidence for altered M1 organisation and function in chronic pain.

Methods and analysis: Systematic review and metaanalysis. We will search electronic databases with predetermined search terms to identify relevant studies and evaluate the studies for inclusion and risks of bias. Two independent reviewers will extract data. Any disagreement will be resolved through a third reviewer. Cross-sectional or prospective studies published in English before May 2015 that investigate M1 organisation and function in chronic pain will be included if they meet the eligibility criteria. Primary outcomes will include M1 cortical excitability, spatial cortical representation, the function of inhibitory and facilitatory intracortical networks, cortical reactivity and cortical glucose metabolism. Clinical measures such as pain and disability will be included where the correlation with the primary outcomes of M1 organisation and function were investigated in the included studies.
\end{abstract}

Ethics and dissemination: This systematic review does not require ethical approval. The results of this review will be submitted for peer-reviewed publication regardless of outcome and will be presented at relevant conferences.

Trial registration number: Our systematic review protocol was registered with the International Prospective Register of Systematic Reviews (PROSPERO; registration number CRD42015014823).

\section{INTRODUCTION}

Musculoskeletal disorders are a common cause of disability and result in significant social and economic costs. ${ }^{1}$ An estimated $10 \%, 5 \%$ and $4 \%$ of the global population with low back pain (LBP), neck pain and knee osteoarthritis, respectively, live with
Strengths and limitations of this study

- Altered organisation and function of primary motor cortex (M1) is implicated in chronic pain. However, to the best of our knowledge, this is the first systematic review of M1 changes across multiple chronic pain conditions.

- Two independent reviewers will assess articles for inclusion and conduct data extraction and risk of bias assessment.

- Data analyses will include meta-analyses where appropriate, as well as subgroup and sensitivity analyses.

disability ${ }^{1}$ with pain being the main symptom of musculoskeletal disorders, especially in the chronic stage. In the USA, nearly $30 \%$ of the adult population live with pain. ${ }^{2}$

Movement dysfunction associated with pain is commonly observed in the clinic and is a key focus of rehabilitation. For instance, when musculoskeletal pain is present, deficits in force production, amplitude and speed of movement, muscle coordination and postural control are reported. ${ }^{3-5}$ Despite this, the physiological basis and clinical relevance of movement dysfunction in pain is poorly understood. There is considerable debate regarding the type, quantity and timing of movement-based treatments, if any, needed to effectively target motor dysfunction in persistent musculoskeletal pain disorders. ${ }^{6-8}$

The primary motor cortex (M1) is a key driver of motor output and may therefore contribute to movement dysfunction in pain, making it a potential target for therapy. There is emerging evidence of altered M1 organisation and function across a range of chronic pain conditions. For example, M1 topographical representations generated using transcranial magnetic stimulation (TMS) show greater overlap and a reduced 
number of discrete peaks in chronic low back ${ }^{9-11}$ and elbow pain, ${ }^{12}$ and these changes are associated with pain severity and/or motor dysfunction. Similarly, there is evidence for increased signal with movement of the affected hand in complex regional pain syndrome (CRPS) using functional MRI ${ }^{13}$ and evidence of reduced GABAergic and glutamatergic M1 function in fibromyalgia that is associated with fatigue. ${ }^{14}$

To our knowledge, only one published systematic review has investigated M1 organisation and function in chronic pain, and this was restricted to CRPS. ${ }^{15}$ That review revealed limited evidence of bilateral M1 disinhibition in CPRS of the upper limb. ${ }^{15}$ However, it is unknown whether similar alterations in M1 are present in other forms of chronic pain. Indeed, one previous study has suggested that M1 disinhibition may occur in chronic neuropathic but not chronic nociceptive pain. ${ }^{16}$ This review will be the first to systematically and critically evaluate the evidence for altered M1 organisation and function, across a range of measurement tools, in chronic pain conditions of neuropathic and nonneuropathic origin. Understanding how M1 organisation and function is altered in chronic pain is essential to inform the design and testing of treatment strategies that seek to target M1 in pain.

Here, we present the protocol for a review that aims to evaluate the evidence for altered M1 organisation and function in chronic pain conditions of neuropathic or non-neuropathic origin. This protocol is prepared according to the Preferred reporting items for systematic review and meta-analysis protocols (PRISMA-P) guidelines. ${ }^{17}$ The protocol was registered with the International Prospective Register of Systematic Reviews (PROSPERO; registration number CRD42015014823).

\section{METHODS AND ANALYSIS \\ Review question}

What is the evidence for altered M1 organisation and function in chronic pain conditions of neuropathic and non-neuropathic origin?

\section{Search strategy}

The methods for this systematic review have been developed according to the MOOSE Guidelines for Meta-Analyses and Systematic Reviews of Observational Studies. ${ }^{18}$ The search strategy will be implemented in two stages.

1. Searches will be conducted in PubMed, MEDLINE, EMBASE, PsychINFO and CINAHL databases to identify relevant literature. Key words and medical subject headings $(\mathrm{MeSH})$ related to chronic pain, neuroimaging and the brain will be used; for example: chronic pain, positron emission tomography, functional magnetic resonance imaging, BOLD contrast, Electroencephalogra*, Magnetoencephalogra*, transcranial magnetic stimulation, motor cortical and sensorimotor cortex. The full search terms are listed in online supplementary appendix 1 . The combination of chronic pain, neuroimaging and brain search terms will be used in varying combinations to identify relevant literature. Search strategies will be customised to suit each database. The main search strategy is included in online supplementary appendix 1 .

2. The reference lists of eligible articles and relevant reviews will be manually searched for additional articles.

\section{Type of participants}

Participants should be adults (aged over 18 years) experiencing chronic, musculoskeletal pain of neuropathic or non-neuropathic origin. Neuropathic pain is defined as "pain caused by a lesion or disease of somatosensory nervous system'. ${ }^{19}$ Non-neuropathic pain is defined as pain without an identifiable lesion or disease of the somatosensory nervous system. ${ }^{20}$ Studies investigating visceral or cancer pain will be excluded. No restriction is placed on the sex of participants. The duration of pain experienced by participants should be greater than 3 months as this duration is commonly defined as the chronic phase of pain. ${ }^{21}$ Cross-sectional or prospective studies will be included in the initial search if they meet the eligibility criteria. Prospective studies including case-control and randomised controlled trials will only be included if their baseline data provide information relevant to the review objective.

\section{Inclusion criteria}

1. Full-text studies, including in press or accepted studies, published in English prior to May 2015.

2. Studies conducted on adult humans with chronic non-neuropathic or neuropathic pain.

3. Studies that investigate the organisation and/or function of the M1 (regardless of the anatomical or functional definition used) with the following techniques: TMS, MRI, positron emission tomography, EEG and magnetoencephalography.

4. Studies including data from a healthy control group.

\section{Exclusion criteria}

1. Studies including participants with chronic pain not of musculoskeletal origin, for example, pain associated with spinal cord injury, stroke, cancer or visceral pain.

2. Studies that do not include a healthy control group or that use the unaffected limb or body side as a control. It is recognised that widespread symptoms remote from the original injury site can be observed in chronic pain. ${ }^{22}$ Thus, using an unaffected limb or body side as a comparison is not considered an appropriate control.

\section{Primary outcomes}

Eligible studies should report one of the following measurements of M1 organisation and/or function: cortical excitability, spatial representations, inhibitory or 
facilitatory intracortical networks, reactivity and/or glucose metabolism as outcomes for analysis in this review. Clinical measures such as pain and disability will be included where these are correlated with the primary outcomes of M1 organisation and function.

\section{Data management}

Two reviewers will independently evaluate the title and abstract of all studies identified through the search against the inclusion and exclusion criteria. Any duplicate studies will be removed. The full text of all eligible studies will then be retrieved. EndNote X7 will be used during the review process to avoid duplicating references. If the reviewer is uncertain about the eligibility of any study, its full text will be obtained for further information. An additional reviewer will be consulted should there be any uncertainty or disagreement of the eligibility of studies. Excluded studies and the reasons for exclusion will be recorded.

\section{Data extraction}

A customised data extraction form (see online supplementary appendix 2) will be piloted on two studies not directly related to this review, and then used to extract data. Two independent reviewers will conduct data extraction. Any disagreements will be resolved through a third reviewer. The following data will be extracted: (1) participant-specific data such as condition, duration and severity of chronic pain, sample size in each group, sex and age; (2) neurophysiological methods and outcomes, specifics of the investigative model such as type and location of stimulation, how M1 was anatomically or functionally defined, neuroimaging findings in M1 excitability, representation, reactivity and glucose metabolism; (3) pain scores. Other outcome measurements such as quantitative sensory tests and movement dysfunction will be extracted if they are correlated with the primary outcomes. If data are missing, authors will be contacted a maximum of three times, after which the data will be considered irretrievable.

\section{Risk of bias (quality) assessment}

To assess the risk of bias of the included studies, we will use the STROBE statement for cross-sectional and cohort studies (see online supplementary appendix 3) and items relevant to case-control studies from the Cochrane Collaboration tool for assessing the risk of bias. ${ }^{23-25}$ Methodological quality pertaining directly to the use of TMS will be assessed via a TMS methodological checklist (see online supplementary appendix 4). ${ }^{26}$ Two independent reviewers will undertake the assessment of risk of bias and methodological quality. Any disagreement will be resolved by a third reviewer.

\section{Strategy for data synthesis}

A quantitative synthesis is planned to aggregate the data from all types of chronic pain conditions. Parameters such as cortical excitability (resting or active motor thresholds, intracortical inhibition, intracortical facilitation), spatial representation (map volume, BOLD response), M1 reactivity or M1 glucose metabolism will be pooled to perform separate meta-analyses using OpenMetaAnalyst. Cohen's d effect sizes will be used to analyse effect estimates: $\mathrm{d} \leq 0.2$ is small, 0.5 represents medium, $\geq 0.8$ is considered large ${ }^{27}$ Data will be pooled for an outcome by using a random-effects model if data from at least two studies addressing that outcome are accessible. The $\chi^{2}$ test will be used to identify statistically significant heterogeneity, and statistically significant heterogeneity will be considered existent when $\chi^{2} p<0.10$. The $\mathrm{I}^{2}$ statistic will be used to evaluate the degree of heterogeneity. Substantial heterogeneity will be considered existent when $\mathrm{I}^{2}>50 \% .{ }^{28}$ All data will be presented as effect estimates (with 95\% CIs). Where quantitative synthesis of the extracted data is not appropriate, a narrative synthesis will be used to summarise the study findings about functional and structural changes of M1. ${ }^{17}$

\section{Analysis of subgroups or subsets}

Where significant heterogeneity is found, we will conduct subgroup analysis according to the type of pain conditions (LBP, CRPS, fibromyalgia, peripheral neuropathic pain or peripheral tendinopathy), duration of pain, sex of participants and type of treatment participants were receiving at the time cortical data were collected.

\section{Sensitivity analysis}

The included studies will be given a score when assessing their methodological quality. For example, studies will score one point if they meet the criteria of 1 of the 22 items from the STROBE statement, hence a maximum 22 points can be scored. The median value of the overall scores of eligible studies will be used as the cut-off point to divide the studies into either the low or high risk of bias group. We will then examine the influence of including studies at high risk of bias by running the analysis with those studies excluded.

Twitter Follow Siobhan Schabrun at @DrSMSchabrun

Contributors W-JC, NEO, EB, LSC, MBL and SMS were each involved in the conception, design, writing and editing of the study protocol. The final protocol was approved by W-JC, NEO, EB, LSC, MBL and SMS.

Funding W-JC, MBL and SMS received salary support from the National Health and Medical Research Council of Australia.

Competing interests None declared.

Patient consent Obtained.

Provenance and peer review Not commissioned; externally peer reviewed.

Open Access This is an Open Access article distributed in accordance with the Creative Commons Attribution Non Commercial (CC BY-NC 4.0) license, which permits others to distribute, remix, adapt, build upon this work noncommercially, and license their derivative works on different terms, provided the original work is properly cited and the use is non-commercial. See: http:// creativecommons.org/licenses/by-nc/4.0/ 


\section{REFERENCES}

1. Vos $T$, Flaxman AD, Naghavi M, et al. Years lived with disability (YLDs) for 1160 sequelae of 289 diseases and injuries 1990-2010: a systematic analysis for the Global Burden of Disease Study 2010. Lancet 2012;380:2163-96.

2. Krueger $A B$, Stone $A A$. Assessment of pain: a community-based diary survey in the USA. Lancet 2008;371:1519-25.

3. Lund JP, Donga R, Widmer CG, et al. The pain-adaptation model: a discussion of the relationship between chronic musculoskeletal pain and motor activity. Can J Physiol Pharmacol 1991;69: 683-94.

4. Grimstone SK, Hodges PW. Impaired postural compensation for respiration in people with recurrent low back pain. Exp Brain Res 2003;151:218-24.

5. Hodges PW, Moseley GL, Gabrielsson A, et al. Experimental muscle pain changes feedforward postural responses of the trunk muscles. Exp Brain Res 2003;151:262-71.

6. Aladro-Gonzalvo AR, Araya-Vargas GA, Machado-Díaz M, et al. Pilates-based exercise for persistent, non-specific low back pain and associated functional disability: a meta-analysis with meta-regression. J Bodyw Mov Ther 2013;17:125-36.

7. Gross A, Kay TM, Paquin JP, et al. Exercises for mechanical neck disorders. Cochrane Database Syst Rev 2015;1:CD004250.

8. Hayden JA, van Tulder MW, Malmivaara A, et al. Exercise therapy for treatment of non-specific low back pain. Cochrane Database Syst Rev 2005;(3):CD000335.

9. Tsao H, Galea MP, Hodges PW. Reorganization of the motor cortex is associated with postural control deficits in recurrent low back pain Brain 2008;131(Pt 8):2161-71.

10. Tsao H, Danneels LA, Hodges PW. ISSLS prize winner: smudging the motor brain in young adults with recurrent low back pain. Spine 2011;36:1721-7.

11. Schabrun SM. Low back pain: new insights and implications for rehabilitation. World Congress of Low Back and Pelvic Pain; 19 October 2013,Dubai, United Arab Emirates.

12. Schabrun SM, Hodges PW, Vicenzino B, et al. Novel adaptations in motor cortical maps: the relation to persistent elbow pain. Med Sci Sports Exerc 2015;47:681-90.

13. Maihöfner $\mathrm{C}$, Baron $\mathrm{R}$, DeCol R, et al. The motor system shows adaptive changes in complex regional pain syndrome. Brain 2007;130(Pt 10):2671-87.

14. Mhalla A, de Andrade DC, Baudic S, et al. Alteration of cortical excitability in patients with fibromyalgia. Pain 2010;149: 495-500.
15. Di Pietro F, McAuley JH, Parkitny L, et al. Primary motor cortex function in complex regional pain syndrome: a systematic review and meta-analysis. J Pain 2013;14:1270-88.

16. Schwenkreis $P$, Scherens A, Rönnau AK, et al. Cortical disinhibition occurs in chronic neuropathic, but not in chronic nociceptive pain. BMC Neurosci 2010;11:73.

17. Shamseer L, Moher D, Clarke M, et al. Preferred reporting items for systematic review and meta-analysis protocols (PRISMA-P) 2015: elaboration and explanation. BMJ 2015;349:g7647.

18. Stroup DF, Berlin JA, Morton SC, et al. Meta-analysis of observational studies in epidemiology: a proposal for reporting. Meta-analysis Of Observational Studies in Epidemiology (MOOSE) group. JAMA 2000;283:2008-12.

19. Merskey H, Bogduk N, Taxonomy IAftSoPSo. Classification of chronic pain: descriptions of chronic pain syndromes and definitions of pain terms. 2nd edn. Seattle: IASP Press, 1994.

20. Picavet HS, Schouten JS. Musculoskeletal pain in the Netherlands: prevalences, consequences and risk groups, the DMC(3)-study. Pain 2003;102:167-78.

21. Ostelo RW, van Tulder MW, Vlaeyen JW, et al. Behavioural treatment for chronic low-back pain. Cochrane Database Syst Rev 2005;(1):CD002014

22. Woolf CJ. Central sensitization: implications for the diagnosis and treatment of pain. Pain 2011;152(3 Suppl):S2-15.

23. von Elm E, Altman DG, Egger M, et al. The Strengthening the Reporting of Observational Studies in Epidemiology (STROBE) statement: guidelines for reporting observational studies. PLoS Med 2007;4:e296.

24. Parkitny L, McAuley JH, Di Pietro F, et al. Inflammation in complex regional pain syndrome: a systematic review and meta-analysis. Neurology 2013;80:106-17.

25. von Elm E, Altman DG, Egger M, et al. The Strengthening the Reporting of Observational Studies in Epidemiology (STROBE) Statement: guidelines for reporting observational studies. Int J Surg 2014;2:1495-9.

26. Chipchase L, Schabrun S, Cohen L, et al. A checklist for assessing the methodological quality of studies using transcranial magnetic stimulation to study the motor system: an international consensus study. Clin Neurophysiol 2012;123:1698-704.

27. Cohen J. Statistical power analysis for the behavioural sciences. 2nd edn. Hillsdale, NJ: Lawrence Erlbaum, 1998.

28. Higgins J, Green S. Cochrane handbook for systematic reviews of interventions version 5.1.0 [updated March 2011]. The Cochrane Collaboration, 2011. 